\title{
A Hybrid Multilevel Approach for Aeroelastic Optimization of Composite Wing-box
}

\author{
Paolo Gasbarri ${ }^{1}$, Leonardo D. Chiwiacowsky ${ }^{2}$, Haroldo F. de Campos Velho ${ }^{3}$ \\ 1: Università degli Studi di Roma - "La Sapienza" \\ Via Eudossiana, 18 - 00184 Rome, Italy \\ 2: Universidade do Vale do Rio dos Sinos (UNISINOS) \\ Av. Unisinos, 950 - 93022-000, São Leopoldo/RS, Brazil \\ 3: Instituto Nacional de Pesquisas Espaciais (INPE) \\ Av. dos Astronautas, 1758 - 12227-010, São José dos Campos/SP, Brazil \\ E-mail: ${ }^{1}$ paolo.gasbarri@uniroma1.it, ${ }^{2}$ Idchiwiacowsky@unisinos.br, ${ }^{3}$ haroldo@lac.inpe.br
}

\section{Abstract}

The quest for finding optimum solutions to engineering problems has existed for a long time. In the last decade several optimization techniques have been applied to structural design of wing composite structures. Generally many of these proposed procedures have dealt with different disciplines such as aerodynamics, structures, or dynamics separately. However an aeronautical design process is multidisciplinary involving strong couplings and interactions between, for instance, aerodynamics, dynamics, flight mechanics and structures. Generally the main problem in a multidisciplinary aircraft design is the development of an efficient method to integrate structures or structural properties, which can be considered both as "global" and "local" design variables. This paper describes an integrated aerodynamic/dynamic/structural optimization procedure for a composite wing-box design. The procedure combines an aeroelastic optimization of a composite wing based on a general purpose optimizer such as the Sequential Quadratic Programming (SQP) and a composite optimization using Genetic Algorithm (GA). Both the optimization are implemented through a multilevel decomposition technique.

\section{Keywords: Structural Optimization, Multilevel Optimization, Genetic Algorithm.}

\section{Introduction}

A wing composite structure is composed of a large number of panels, which have to be designed simultaneously to obtain optimum structural design. Usually the design of each panel requires a large number of design variables to describe its geometry, ply composition and laminate stacking sequences. In view of these considerations, designing all the panels simultaneously constitutes an extremely large optimization problem, requiring also a very detailed structural model of the entire wing, that appears to be beyond the present computational capabilities. In general the wing box design process is a multidisciplinary one, involving couplings and interactions among several disciplines such as aerodynamics, structural analysis, dynamics, and aeroelasticity. The optimization problem, related to a wing-box design, involves multiple objectives and constraints pertaining to the design criteria associated with each one of these disciplines. These couplings and interactions can be explored by optimization procedure if all the disciplines are accounted for simultaneously rather than sequentially $[1,2]$. Therefore, the development of multidisciplinary design optimization (MDO) techniques, in which different disciplines and design parameters are coupled into a closed loop numerical procedure, seems appropriate to address such a complex problem. For a wing box optimization it is very important to have an efficient method to integrate structures or structural properties into the procedure itself. Usually, structural properties are included in one of two ways, either as a local design variable (indirectly affecting the response of the wing) or as global design variable (directly affecting the response of the wing). When local design variables are used, the detailed dimensions of the structural members at one or more wing-span locations are used to generate structural properties. When global design variables are used, structural properties are the design variables. Both types of design variables have limitations. Using local variables can lead to a large number of parameters that can be computationally expensive. Furthermore this choice is not practical from a traditional design point of view in which chord, stiffness, and mass distribution along the wing-span are determined and then a structure is designed which matches these distributions. Using global quantities such as stiffness 
and mass properties in optimization procedure has also disadvantages. When, for example, flexural and torsional stiffness distributions are used as design variables, they are treated as independent quantities. In reality, these stiffnesses are not independent, and no guarantees can be given that a set of thicknesses, section geometries can be found that will simultaneously give these stiffnesses.

In the last years many researchers in aerospace industries as well as in aerospace research centers have introduced the multilevel decomposition technique in MDO. The idea is to incorporate aerodynamics, dynamics and structures in an integrated optimization procedure using both local and global design variables. With a multilevel decomposition approach [3, 4] a large complex optimization problem is broken into a hierarchy of smaller optimizations subproblems. This hierarchy can be thought of as a level of increasing details. At the upper level, the subproblem is formulated in terms of global quantities which describe the overall behavior of the entire system. On the lower level, the subproblems are stated in terms of local quantities and local constraints which have only a small impact on the entire system. Each subproblem uses local design variables to reduce the violation of constraints which are unique to that subproblem. The coupling between the upper level subproblems and the lower ones is preserved through a coordination procedure such as that described in [5]. This coupling represents a dialogue between the levels that upon the convergence establishes compatibility between the two levels. Fig. 1(a) (from reference [2]) illustrates a two level optimization procedure.

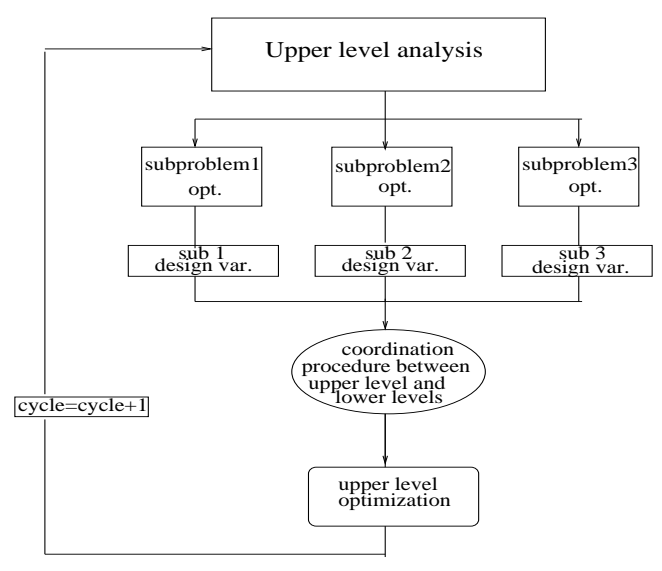

(a)

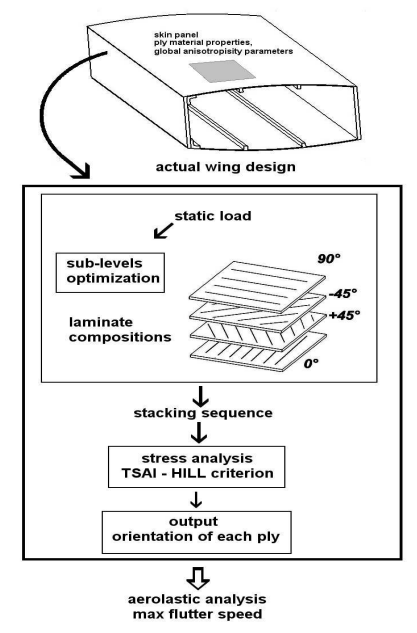

(b)

Figure 1: (a) General multilevel optimization procedure with two levels; (b) Wing-box optimization criterion.

As shown in Fig. 1(a) the analysis proceeds from the upper to the lower level while the optimization proceeds from the lower to the upper level. As a first step, the upper level analysis initializes all the global quantities and responses and provides information to each of the lower level subproblem. In a second step each individual lower level optimization problem is performed. These procedures must reduce local constraint violations as much as possible and must provide information to the coordination procedure. In a third step the upper level optimization occurs. We must consider these three steps as one cycle of the entire process. Convergence occurs when all the constraints (both upper level and lower level) are satisfied and the upper level objective function is minimized.

For a composite wing structure design it is also possible to consider a two level optimization procedure. At a global level, individual wing-panels are modeled without many details, and few design variables can be used to define each panel. Global constraints, such as stress or strain limits and also maximum flutter or divergence aeroelastic velocities can be enforced for instance using finite element structural optimization programs. These global parameters obtained by such optimization are used as input to panel design optimization programs. These programs obtain detailed geometry and ply compositions for each panel individually, enforcing for example buckling constraints and/or various strength failure constraints.

Usually the integration of the two levels, the local and global one, is difficult and must be handled mostly 
by ad-hoc methods, which are awkward and suboptimal.

In this work a method to characterize composite panels of a wing box structure will be presented. A two level approach will be also used for a MDO; in the first one, very few global quantities, such as the degree of anisotropicity of the material will defined for the global aeroelastic optimization process, while, at the second level optimization process, an efficient procedure will be introduced to obtain the ply composition and the stacking sequences for the laminate composite plates.

3.1. A two level optimization approach for aeroelastic optimization

The aeroelastic optimization here presented is relevant to the determination of the orientation of different layers which constitute the composite panels of a wing box structure, that realizes the maximum flutter speed. Strength constraints for the composite panels are also applied. For the conventional approach we can address the problem directly by using, as design variables, the ply orientations of the layers at one or more span locations along the wing.

By using a multilevel approach the aeroelastic optimization problem can be decomposed into one subproblem affecting the global response of the wing and several independent subproblems affecting portions of the wing. For the case under concern the flutter velocity is used to describe the global response of the wing. Quantities such as stresses, due to applied loads if any, are instead detailed response quantities since only a portion of the wing must be analyzed to obtain these ones. Therefore a two level decomposed wing optimization problem can be defined as shown in Fig. 1(b).

The upper level optimizes the wing by changing global quantities, in this case defined through some parameters related to the degree of anisotropicity of the material (see Sections 5.2 and 5.3). These parameters are treated as independent quantities even if they are not. The reconciliation between these quantities and the real design variables is done on the lower level. The lower level consists of independent subproblems at different stations along the wing, which optimize the lay-up angles to reconcile the upper level independent variables. This reconciliation is improved further by a set of upper level coordination constraints (see Section 5.4).

\section{Governing equations}

4.1. Wing structure

In this work the wing is assumed to consist of a wing-box stiffened composite structure $[6,7]$. A plate model is used to represent the wing box. The planform geometry of this plate is assumed to be trapezoidal, with arbitrary sweep angle $\Lambda$, with respect to the wind direction, and a non dimensional non-orthogonal coordinate system $(u, \eta)$ is adopted as illustrated in Fig. 2(a) and reported in Eq. (1).

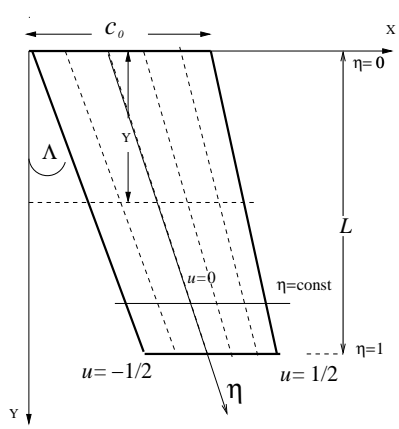

(a)

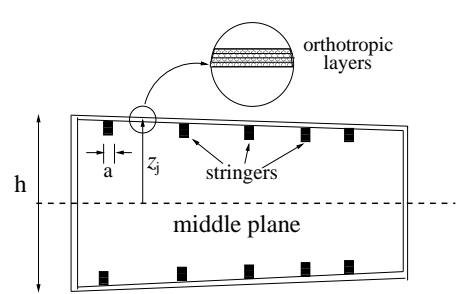

(b)

Figure 2: (a) Wing planform geometry; (b) Wing-box section.

$$
\left\{\begin{array}{l}
\eta(X, Y)=Y / L ; \quad 0 \leq \eta \leq 1 \\
u(X, Y)=-\frac{1}{2}+\frac{X-\tan \Lambda Y}{c} ; \quad-1 / 2 \leq u \leq 1 / 2
\end{array}\right.
$$


A generic point $M$ of the wing is detected by: $\eta(M)=A_{0} M_{0} / L$ and $u(M)=O M / C_{0} C_{1}$. So coordinate lines of the new system are the horizontal lines $\eta=$ const. (coinciding with the lines $Y=$ const. of the first system) and the dotted lines $u=$ const. Furthermore, the orthogonal and nonorthogonal systems are connected by the following differentiation rule:

$$
\frac{\partial \phi}{\partial X}=\frac{1}{c} \frac{\partial \phi}{\partial u} ; \quad \frac{\partial \phi}{\partial Y}=\frac{1}{L} \frac{\partial \phi}{\partial \eta}-\frac{\theta}{c} \frac{\partial \phi}{\partial u} .
$$

As far as the elastic properties are concerned, they are described by a matrix $\mathbf{H}$ (relevant to the material distribution along the thickness $z$ of the wing). So the constitutive equations can be expressed as $\{\sigma\}=[\mathbf{H}]\{\epsilon\}$ where the coefficient $H_{j k}=H_{j k}(u, \eta, z)$.

\subsection{General Considerations and Kinematics}

The wing is assumed to be of uniform cross section, although the model is capable of modeling also non uniform one. The presence of ribs, insuring the transfer of shear stress between the upper and lower skins is assumed in order to neglect the shear deformations of the cross-sections (see Fig.2(b)). The upper and lower skins are made of several layers that are assumed to have the same mechanical properties with different orientations; furthermore the upper and lower skins and the corresponding layers are assumed to be symmetric about the middle plane of the wing. The anisotropic plate bending stiffness matrix $\mathbf{D}$ is evaluated by means of the classical laminated theory. Stringers laying span-wise on $u=$ const. lines and symmetrically placed with respect to the middle plane of the wing, are represented as axial elements discretely attached to the skins.

As it is well known, in a $z$-symmetric plate, the only elastic displacement to be taken into consideration is the midplane transversal displacement $w=w(X, Y)=w(u, \eta)$, if shear flexibility in the $x z-$ and $y z-$ planes (not necessary rotary inertia) is neglected. In this work we will use a special formulation of finite element method to describe the aeroelastic equations of the composite wing [8]. The kinematics of the plate is described as follows:

$$
\epsilon_{x}(z)=z\left(\partial^{2} w / \partial x^{2}\right) ; \quad \epsilon_{y}(z)=z\left(\partial^{2} w / \partial y^{2}\right) ; \quad \gamma_{x y}(z)=2 z\left(\partial^{2} w / \partial x \partial y\right) .
$$

Thus the operation $\int_{-h / 2}^{h / 2} z \sigma d z$, yields the elasticity equation for the plate:

$$
\mathbf{M}=\mathbf{D}[\mathbf{2}] \mathbf{\Theta},
$$

where $\mathbf{M}^{T}=\left[\begin{array}{lll}M_{x} & M_{y} & M_{x y}\end{array}\right]$ is the moment-vector, $\boldsymbol{\Theta}^{T}=\left[\partial^{2} w / \partial x^{2} ; \partial^{2} w / \partial y^{2} ; \partial^{2} w / \partial x \partial y\right]$ is the curvature vector, $\mathbf{D}$ is the plate stiffness matrix, whose generic component is:

$$
D_{j k}=D_{j k}(u, \eta)=\int_{-h_{t}}^{h_{t}} z^{2} H_{j k}(u, \eta, z) d z ; \quad h_{t}=h(u, \eta) / 2,
$$

(so as to include also the case of $z$-varying elasticity: in case of stepwise variation of $\mathbf{H}$, the integral must proceed stepwise). Finally $[\mathbf{2}]$ is the matrix $\operatorname{diag}[1,1,2]$, introduced so as to cope with the expression of $\gamma_{x y}$. The kinematics of the stringer is described through their axial displacements $v(\eta)$, whereas the elasticity equation for the string is assumed as follows:

$$
N=E A \frac{d v}{d y}
$$

being $E$ the Young's module and $A$ the cross sectional area of the stringer.

\subsection{Finite Element Method}

A modified finite element method [8] is used to obtain the total energy $\mathcal{E}$ associated to the analytical model. This energy is as follows:

$$
\mathcal{E}=(\mathcal{U}-\mathcal{T}+\mathcal{L}),
$$

where $\mathcal{U}$ and $\mathcal{T}$ denote the strain energy and the kinetic energy of the system and $\mathcal{L}$ denote the work done by the external loads (e.g. aerodynamic loads). Note that aerodynamic loads are nonconservative 
body and surfaces forces. These forces are not derivable from a potential function and they are regarded as known function of the time and position.

The modified finite element method is based on assuming a span-wise finite element discretization of the wing elastic displacement $w$, while a power expansion is assumed chord-wise, the relevant kinematics is as follows:

$$
w(u, \eta)=w_{0}(\eta)+u w_{1}(\eta)+u^{2} w_{2}(\eta)+\ldots+u^{N} w_{N}(\eta) .
$$

With this representation the displacement $w$ is assumed differentiable along the chord as many times as necessary, furthermore $\mathbf{w}_{k}(k=0, \ldots, N)$ and its derivative $\partial w_{k} / \partial \eta$ are assumed continuous. This assumption has the advantage to provide the analyst, directly, global chord-wise quantities such as flexural displacements $w_{0}$, torsional rotations $w_{1}$, mean-line curvature $w_{2}$ etc., which are meaningful from the aerodynamic stand point.

For the sake of brevity all the analytical details relevant to this methodology and its use to evaluate via the classical FEM theory the mass matrix, the stiffness matrix and consequently the dynamic behavior of a composite swept wing are omitted and can be found in [6]. Suffice here to say that the final form is as follows:

$$
\mathbf{M} \ddot{\mathbf{X}}+\mathbf{K X}=\mathbf{F}_{e x t}
$$

where $\mathbf{M}$ and $\mathbf{K}$ are the mass matrix and the stiffness matrix of the structure and $\mathbf{F}_{\text {ext }}$ is the vector of the external forces.

\subsection{Aerodynamic model}

For the present study it is necessary to have an aerodynamic tool that can adequately describe the aerodynamic unsteady loads acting on the wing. One of the most popular method that can be used to determine aerodynamic forces in subsonic compressible flow is the Kernel Function Method (KFM), which is able to characterize the aerodynamic pressure in some wing colocated points. One of the major advantages of KFM is that few colocated points (control points) are able to well describe the pressure distribution on the wing, so that few aerodynamic degrees of freedom are needed [9]. For the sake of simplicity we will consider here the case of quasi-steady aerodynamics which means that: $(i)$ aerodynamic derivatives do not have a time variation, and ( $i i)$ they can be evaluated by using the steady part of KFM. The equation of KFM here considered is as follows:

$$
\frac{w(x, y)}{U_{\infty}}=\frac{1}{8 \pi q_{\infty}} \oint_{\Sigma} G\left(x, y, \bar{x}, \bar{y} ; M_{\infty}\right) \Delta p(\bar{x}, \bar{y}) d \Sigma,
$$

where $(x, y)$ and $(\bar{x}, \bar{y})$ are the coordinates of a generic control point and of the doublet singularity point respectively and $\oint$ here denotes Hadamard's principal value of the integral. The idea of the KFM is to approximate the unknown pressure distribution $\Delta p(\bar{x}, \bar{y})$ in chord-wise and span-wise directions by prescribed functions with unknown coefficients. The right side of Eq. (10) is then evaluated for as many control points $P_{m n}$ as the unknown coefficients and is equated to the known downwash angle at each control point. A set of simultaneous equations is thus obtained which may be solved to determine the unknown constants.

Then the constants are used to define the pressure distribution. The final expression of the pressure distribution reads:

$$
\Delta \bar{p}(v, \eta)=8 \pi q_{\infty} \boldsymbol{\Gamma}^{T}(v, \eta) \mathbf{A}^{-1} \mathbf{J}
$$

where $\boldsymbol{\Gamma}^{T}(v, \eta)$ is the aerodynamic shape function vector, $\mathbf{A}^{-1}$ is the inverse matrix of the algebraic solving system (depending on Mach number $M_{\infty}$ ), and $\mathbf{J}$ is the vector of down-washes evaluated at the aerodynamic control points $P_{m n}[9]$.

\subsection{Aeroelastic equations}

The relationship between aerodynamics and structure can be found by evaluating the work performed by aerodynamic forces on the generalized displacements:

$$
\mathcal{L}_{j}=\int_{S_{j}} w_{j}(u, \eta) \Delta p_{j}(u, \eta) d u d \eta
$$


where $w_{j}$ is the displacement of the $j$-th wing element of area $S_{j}$ (if the domain of integration is discretized according to the FEM rules) and $\Delta p_{j}(u, \eta)$ is the pressure distribution given by Eq. (11). The vector form of the aerodynamic forces acting on the wing box to be used with FEM formulation reads as follows:

$$
\mathbf{F}_{\text {ext }}=q_{\infty} \mathbf{R} \mathbf{J}
$$

being $\mathbf{R}$ the so-called aerodynamic matrix.

The vector of down-washes $\mathbf{J}$ can be divided into two parts: the one relevant to geometric incidence $\alpha_{g}$ and the one relevant to elastic incidence $\alpha_{e}$ :

$$
\mathbf{J}=\alpha_{g}+\alpha_{e}
$$

The elastic incidence can be expressed through the deflection of the structural points of finite element discretization: $\alpha_{e}=\mathbf{Q X}$, being $\mathbf{Q}$ the matrix which transforms the structural degrees of freedom into the aerodynamic ones. Combining Eqs. (13) and (14) and substituting into Eq. (9) the final aeroelastic equation is obtained:

$$
\mathbf{M} \ddot{\mathbf{X}}+\mathbf{K X}=q_{\infty} \mathbf{R}\left\{\alpha_{g}+\mathbf{Q X}\right\}=\mathbf{F}_{a e r}
$$

The above equation is the basic formula for studying the aeroelastic phenomena (both static and dynamic) on elastic wings.

Let us now consider the case of flutter analysis which, as well known, studies the stability behavior of the wing under dynamic aerodynamic loads. In particular, flutter conditions are those particular flight conditions (i.e. a particular flight velocity $U_{\infty}$ ) in which a wing structure (with its geometry and mechanical properties) has a dynamic unstable behavior when perturbed from its equilibrium position. As it is well known a very efficient method of solution for investigation of dynamic behavior of the structures is represented by the dynamic response in harmonic time-variation. For this approach the solving equation can be derived from Eq. (15) as follows:

$$
\left(-\omega^{2} \mathbf{M} \overline{\mathbf{X}}+j \omega \mathbf{C} \overline{\mathbf{X}}+\mathbf{K} \overline{\mathbf{X}}\right)=\overline{\mathbf{F}}_{a e r},
$$

where $\overline{\mathbf{F}}_{a e r}$ is the transform of $\mathbf{F}_{a e r}, \overline{\mathbf{X}}$ is the transform of $\mathbf{X}$ in the frequency domain $\omega$ and $\mathbf{C}$ is the matrix of the structural damping here introduced to describe the real dynamic behavior of the structure. Following standard procedure, free vibration frequencies and corresponding eigenmodes of the wing can be obtained neglecting the right term of the above linear dynamic equation. These eigenmodes can be used to define a small subspace of size $\Re^{M_{2}}$ (with $M_{2}$ denoting the number of eigenmodes) where the flutter analysis of the wing can be solved. Let's consider the first $M_{2}$ normal modes of the structure, $\boldsymbol{\Phi}_{r}(x, y)\left(r=1,2, \ldots, M_{2}\right)$, and let $\mathbf{X}=\boldsymbol{\Phi}^{T}(x, y) \mathbf{Y}$, where $\boldsymbol{\Phi}$ is the vector of the $\boldsymbol{\Phi}_{r}$ 's, and $\mathbf{Y}$ is the vector of modal amplitudes. Then the generalized aerodynamic forces vector (e.g the right term in Eq.(15)), as projected on the normal modes, can be written in the following form:

$$
\mathbf{F}_{a e r}=q_{\infty}\left(\mathbf{L}_{A} \mathbf{Y}+\mathbf{L}_{B} \alpha_{g}\right),
$$

where $\mathbf{L}_{A}$ and $\mathbf{L}_{B}$ are the matrices of aerodynamic forces in the subspace $\Re^{M_{2}}$ of the eigenmodes not reported here for the sake of brevity. Defining the matrix $\Omega=\operatorname{diag}\left(\omega_{1}, \omega_{2}, \ldots, \omega_{M_{2}}\right)$, where $\omega_{j}=j$-th circular eigenfrequency of the structure, the aeroelastic Eq. (15) in the frequency domain can be written as follows:

$$
\left[\Omega^{2}-\omega^{2} \mathbf{I}+2 j \omega \Omega \mathbf{C}-q_{\infty} \mathbf{L}_{A}\right] \mathbf{Y}=0
$$

where $\mathbf{I}$ is the unit $M_{2} \times M_{2}$ matrix and $\mathbf{C}$ is the matrix of the structural damping. The right term connected to the geometric incidences $\alpha_{g}$ has been neglected for the case of flutter and/or divergency velocity evaluation. Note that these problems are relevant to the study of the wing-structure stability so the associated system of equations is an homogeneous one. The condition:

$$
\operatorname{det}\left[\Omega^{2}-\omega^{2} \mathbf{I}+2 j \omega \Omega \mathbf{C}-q_{\infty} \mathbf{L}_{A}\right] \mathbf{Y}=0
$$

is the working form of the flutter equation. In fact, Eq. (19) constitutes a system of two equations (real part $=$ imaginary part $=0$ ) which yields the values of $q_{\infty}=q_{c r}$ and of the flutter eigen-frequencies for 
a given wing geometry, flight conditions (i.e $\rho_{\infty}$ and $M_{\infty}$ ), and wing material properties (i.e. flexural stiffness of the wing-box and axial stiffness of the stringers). With Eq. (19) it is also possible to analyze the aeroelastic static response in both subcritical and critical flight regimes.

\section{Wing-Box optimization}

5.1. Composite laminate

The composite laminate design process typically involves the optimization of the following three parameters: (i) Ply (or lamina) material; (ii) Ply thickness, and (iii) Ply orientation.

The optimization of a composite laminate, simultaneously considering the coupling effects of the design parameter mentioned above, is a mathematical challenge in structural optimization procedure. The optimization of the parameter "ply material" is, perhaps, the most complex of all, because of the inherent possibility of designing a hybrid laminate consisting of two or more material types. As far as items (ii) and (iii) are concerned, numerous analytical techniques are available to optimize the ply thickness and orientation of a composite laminate $[10,11]$. The majority of the commercially available structural optimization codes treat ply thickness and orientation as continuous design variable, although recently some codes have been enhanced to handle them as a discrete and/or a continuous variable during the laminate design process [12].

In this paper it has only been considered the optimal orientation problem of a two-dimensional linearly elastic structure, characterized by the following constitutive law *:

$$
\left\{\begin{array}{l}
\sigma_{1} \\
\sigma_{2} \\
\tau_{12}
\end{array}\right\}=\left[\begin{array}{lll}
H_{11} & H_{12} & H_{16} \\
H_{21} & H_{22} & H_{26} \\
H_{31} & H_{32} & H_{36}
\end{array}\right]\left\{\begin{array}{l}
\epsilon_{1} \\
\epsilon_{2} \\
\gamma_{12}
\end{array}\right\}=[\mathbf{H}]_{j}\left\{\begin{array}{l}
\epsilon_{1} \\
\epsilon_{2} \\
\gamma_{12}
\end{array}\right\},
$$

where $\sigma_{1}, \sigma_{2}, \tau_{12}$ are stress components, $\epsilon_{1}, \epsilon_{2}$ and $\gamma_{12}$ are strain components and $\mathbf{H}_{j}$ is the rotated orthotropic stiffness matrix for the $j$-th lamina of the composite laminate. The relationship between rotated stiffness matrix $\mathbf{H}_{j}$ and unrotated orthotropic stiffness matrix $\mathbf{H}_{0}$ (note that in the following work all the layers have been assumed to be made by the same material) can be established through the standard rotation matrix $\mathbf{T}\left(\theta_{j}\right)$ where $\theta_{j}$ is the angle of orientation of the $j$-th lamina with respect to the laminate reference frame:

$$
\mathbf{H}_{j}=\mathbf{T}^{T}\left(\theta_{j}\right) \mathbf{H}_{0} \mathbf{T}\left(\theta_{j}\right),
$$

where $\mathbf{H}_{0}$ and $\mathbf{T}\left(\theta_{j}\right)$ are as follows:

$$
\mathbf{H}_{0}=\left[\begin{array}{lll}
H_{11}^{0} & H_{12}^{0} & 0 \\
H_{12}^{0} & H_{22}^{0} & 0 \\
0 & 0 & H_{66}^{0}
\end{array}\right] ; \quad \mathbf{T}\left(\theta_{j}\right)=\left[\begin{array}{ccc}
c_{\theta}^{2} & s_{\theta}^{2} & -2 c_{\theta} s_{\theta} \\
s_{\theta}^{2} & c_{\theta}^{2} & 2 c_{\theta} s_{\theta} \\
c_{\theta} s_{\theta} & -c_{\theta} s_{\theta} & c_{\theta}^{2}-s_{\theta}^{2}
\end{array}\right]_{j},
$$

where $c_{\theta}=\cos \theta_{j}$ and $s_{\theta}=\sin \theta_{j}$. Expanding Eq. (21) follows:

$$
\mathbf{H}_{j}=\mathbf{H}^{00}+\mathbf{H}^{01} \cos 2 \theta_{j}+\mathbf{H}^{02} \cos 4 \theta_{j}+\mathbf{H}^{10} \sin 2 \theta_{j}+\mathbf{H}^{12} \sin 4 \theta_{j}
$$

where the matrix $\mathbf{H}^{00}$ and the matrices $\mathbf{H}^{l k}(l=0,1$ and $k=0,1,2)$ contain the invariants w.r.t the rotation operation, e.g terms that are not dependent on ply orientation. For the case under concern follows:

$$
\begin{gathered}
\mathbf{H}^{00}=\left[\begin{array}{lll}
U_{1} & U_{4} & 0 \\
U_{4} & U_{1} & 0 \\
0 & 0 & U_{5}
\end{array}\right] ; \quad \mathbf{H}^{01}=\left[\begin{array}{lll}
U_{2} & 0 & 0 \\
0 & -U_{2} & 0 \\
0 & 0 & 0
\end{array}\right] ; \quad \mathbf{H}^{02}=\left[\begin{array}{ccc}
U_{3} & -U_{3} & 0 \\
-U_{3} & U_{3} & 0 \\
0 & 0 & -U_{3}
\end{array}\right] ; \\
\mathbf{H}^{10}=\left[\begin{array}{lll}
0 & 0 & U_{2} / 2 \\
0 & 0 & U_{2} / 2 \\
U_{2} / 2 & U_{2} / 2 & 0
\end{array}\right] ; \quad \mathbf{H}^{12}=\left[\begin{array}{ccc}
0 & 0 & U_{3} \\
0 & 0 & -U_{3} \\
U_{3} & -U_{3} & 0
\end{array}\right] ;
\end{gathered}
$$

where $U_{1}, U_{2}, U_{3}, U_{4}, U_{5}$ are material parameters defined in [14].

\footnotetext{
${ }^{*}$ Note that this constitutive law will be used for calculating the stiffness matrix $\mathbf{K}$ of the wing box structure.
} 
From the expression of the matrix $\mathbf{H}_{j}$, Eq. (23), it is possible to observe that its coefficients are harmonic functions of the orientation angle $\theta$. This is a complicating factor for the optimization process of the composite laminate structure, since design functions have revealed the presence of multiple optima [13].

5.2. Laminate plate theory: local variables vs. global variables

Let us now consider the case of symmetric orthotropic laminate, where the equilibrium equation is given by Eq. (4), the orthotropic plate bending stiffness matrix [D], is defined as follows:

$$
\mathbf{D}=\sum_{j=1}^{N} \frac{\bar{z}_{j}^{3}-\bar{z}_{j-1}^{3}}{3} \mathbf{H}_{j}
$$

where $\bar{z}_{j}=1 / 2\left(z_{j}+z_{j-1}\right)$ is the position of the $j$-th layer, $N=$ number of layers. It is also assumed that $\mathbf{H}_{j}$ is constant from $z_{j-1}$ to $z_{j}$ which is the thickness domain of the ply number $j$.

By substituting expression (23) into Eq. (26) the matrix of bending stiffness $\mathbf{D}$ is obtained as follow:

$$
\mathbf{D}=\mathbf{D}^{00}+\mathbf{H}^{01} \zeta_{3}+\mathbf{H}^{02} \zeta_{1}+\mathbf{H}^{10} \zeta_{4}+\mathbf{H}^{12} \zeta_{2},
$$

where the terms $\zeta_{k}(k=1, \ldots, 4)$ are functions of the orientation of the layers of laminate, of their thickness and of their stacking sequence:

$$
\zeta_{k}=\zeta_{k}\left(\theta_{1}, \theta_{2}, \ldots, \theta_{N} ; z_{0}, z_{1}, \ldots, z_{N-1}\right) ; \quad k=1, \ldots, 4 .
$$

(the expressions of $\mathbf{D}^{00}$ and of functions $\zeta_{k}$ are reported in Appendix A).

By using the decomposition in Eq. (27) it was possible to isolate terms which explicitly depend on the ply orientation from the ones that do not. Note also that the matrix $\mathbf{D}^{00}$ depends only on the ply thickness and on the ply material and do not depend on the ply angle. This decomposition is very important in the view of the optimization process described later.

\subsection{Aeroelastic optimization}

As mentioned in Section 1, the goal of this optimization investigation is to improve the aeroelastic performance of an airplane wing by using composite tailoring, i.e. the determination of the optimal orientation of the composite laminates which maximizes the aerodynamic pressure $q_{\infty}$ following certain composite failure constraint conditions.

Before doing this, let us now introduce global quantities necessary to describe the mechanical behavior of a wing like-plate composite structure, that will be used at the first level of the optimization procedure. By decomposing the flexural stiffness matrix $\mathbf{D}$ as performed in Eq. (27), it is clear that all the angle ply design variables, i.e. the variables $\theta_{j}(j=1, \ldots, N)$, appropriately weighted with terms that depend on the cubic power of their thickness, appear globally in the functions $\zeta_{k}(k=1, \ldots, 4)$.

It is possible now to describe the degree of anisotropicity of the composite laminate only by means of four global terms instead of all the possible combinations of the ply orientations. Although these four functions are not independent from each other, these can be considered as the only parameters (global parameters) that must be taken into account at the first level of the aeroelastic optimization process. By doing this two different effects are obtained: the first one is the reduction in the number of design variables for the optimization of the composite panel, since, at least at this level, different layer orientations do not have to be considered. In fact the stiffness matrix of the composite panels is only function of the $\zeta_{k}$ 's: $\mathbf{D}=\mathbf{D}\left(\zeta_{1}, \zeta_{2}, \zeta_{3}, \zeta_{4}\right)$; and secondly, which is more important from a numerical point of view, the problem to determine the "optimum" of a function which depends harmonically on the design variables $\theta_{j}$ (see Eq. (23)) has been eliminated, which on your turn could lead to find a local optimum instead of the global one.

\subsection{First level optimization}

The first level optimization consists of finding the maximum value of flutter velocity for assigned values of global design variables $\zeta_{k}$ along the wing-span. In the following, due to the mechanical characteristics of the generic layer used in this paper, the matrices containing the terms $U_{2}$, e.g. $\mathbf{H}^{01}$ and $\mathbf{H}^{10}$ in Eq. (27), 
are assumed equal to zero. For this reason, the design variables for the composite panel become only two: $\zeta_{1}$ and $\zeta_{2}$.

The formulation of the problem may be stated as follows:

Find $(\mathcal{X})$ to maximize $\mathcal{F}_{I}(\mathcal{X})$

$$
\text { subject to } \quad g_{k}(\mathcal{X}) \leq 0 ; \text { and } h_{k}(\mathcal{X})=0 ; \quad k=1, \ldots, N_{L} ; \quad \mathcal{X}_{l} \leq \mathcal{X} \leq \mathcal{X}_{u} ;
$$

where $\mathcal{F}_{I}(\mathcal{X})$ is the flutter velocity obtained from Eq. (19), $\mathcal{X}$ is the design variable vector, containing the global design variables in an assigned number $N_{L}$ of wing-span locations; the subscripts $l$ and $u$ represent lower and upper limit on design variables respectively, and $g_{k}$ and $h_{j}$ are inequality and equality constraints respectively as defined in Section 5.6. Notice that once the thicknesses of the layers of composite panels have been assigned, the upper and lower bounds $\mathcal{X}_{l}$ and $\mathcal{X}_{u}$ can be easily determined by using Eq. (36) (see Appendix A).

5.5. Second Level Optimization: from global variables to local variables

This section of the paper describes the lower level analysis and lower level optimization procedure. The purpose of each lower level optimization is to assess whether the composite panel at a given wing-span location can be tailored to provide the stiffness required by the upper level optimization and have the strength to withstand loads calculated by the upper level analysis. The lower levels optimizations can be done in parallel since they are independent. The lower level design variables are the orientation angles $\theta_{j},(j=1, \ldots, N)$ of each ply of the laminate. The objective function is a measure of the difference between the functions $\bar{\zeta}_{1}$ and $\bar{\zeta}_{2}$ required on the upper level and those determined by prescribing the lower design variables $\theta_{k}$ as reported in Appendix A:

$$
\mathcal{F}_{I I}=\frac{\left(\zeta_{1}-\bar{\zeta}_{1}\right)^{2}}{\bar{\zeta}_{1}^{2}+\bar{\zeta}_{2}^{2}}+\frac{\left(\zeta_{2}-\bar{\zeta}_{2}\right)^{2}}{\bar{\zeta}_{1}^{2}+\bar{\zeta}_{2}^{2}}
$$

where barred quantities denote upper level design variables.

As far as the lower level constraints are concerned they can be considered as side constraints:

$$
-90^{\circ} \leq \theta_{j} \leq+90^{\circ} ; \quad j=1, \ldots, N
$$

After the minimization of the objective function (29) the process returns to the upper level for the next optimization cycle. Note that the number of second level optimizations is equal to the number $N_{L}$ of wing-span sections in which the the wing planform will been divided for the first level optimization.

5.6. Coordination between upper and lower levels

The coordination between upper and lower levels is implemented by upper levels constraints. Specifically, these constraints, one for each second level optimization, have been implemented in two different ways: the first one, which is called as direct constraint coordination procedure, consists of imposing the following equality constraints condition:

$$
h_{k}=\mathcal{F}_{I I, k}^{L}=0 ; \quad k=1, \ldots, N_{L},
$$

where $\mathcal{F}_{I I, k}^{L}$ is the most recent value of the $k$-th second level objective function, and $N_{L}$ is the number of the lower levels. The second type of constraint is inherent to the strength of the composite panel, and it can be imposed by using the classical composite failure criteria such as the Tsai-Hill criteria or Tsai-Wu criteria [14].

The Tsai-Hill constraint, which is evaluated at the upper layer of the laminate, has the following form:

$$
g_{k}=\left(\frac{\sigma_{1}}{\sigma_{1}^{*}}\right)^{2}-\left(\frac{\sigma_{2}^{*}}{\sigma_{1}^{*}}\right)\left(\frac{\sigma_{1} \sigma_{2}}{\sigma_{1}^{*} \sigma_{2}^{*}}\right)+\left(\frac{\sigma_{2}}{\sigma_{2}^{*}}\right)^{2}+\left(\frac{\tau_{12}}{\tau_{12}^{*}}\right)^{2}-1 \leq 0 ; \quad k=1, \ldots, N_{L},
$$

where starred quantities $\sigma_{1}^{*}, \sigma_{2}^{*}$ and $\tau_{12}^{*}$ denote the strength values along the Principal Material Directions, whereas no starred quantities are the stresses associated to an external load. It is important to observe that in order to apply the Tsai-Hill constraint relationship it is necessary to evaluate the stresses acting on the layers of the laminate in the Principal Material reference frame. So it is necessary to use 
the results of lower level optimizations, e.g. the angles $\theta_{j}(j=1, \ldots, N)$ of the layers, to rotate the stress components from the wing reference frame to the lamina reference frame. As far as the stress $\sigma_{1}$, $\sigma_{2}$ and $\tau_{12}$ are concerned they are in this work evaluated through the static aeroelastic equation:

$$
\left(\mathbf{K}-q_{\infty}^{S} \mathbf{R} \mathbf{Q}\right) \mathbf{X}=q_{\infty}^{S} \mathbf{R} \mathbf{Q}\left\{\alpha_{g}\right\}
$$

when the dynamic pressure $q_{\infty}^{S}$ and the geometric incidence vector $\alpha_{g}$ are assigned. Here $\mathbf{X}$ is the vector of the elastic generalized displacement from which through classical FEM rules it is possible to evaluate interlaminate strains and stress.

\section{Optimization and numerical results}

A gradient based optimization technique based on Sequential Quadratic Programming (SQP) and on the method of feasible directions [15] has been used coupled with a stochastic approach represented by the Genetic Algorithm (GA) [16, 17]. The first one has been employed on the solution of the optimization problem at the upper level, i.e. find the maximum flutter velocity, and the latter has been used to solve the different optimization problems at a the lower level related in the finding of the laminate composite stacking sequence for some prescribed wing-span locations. The GA method has been chosen to solve these problems due to two principal reasons: make possible a more comprehensive scan on the search space and avoid been trapped at local optima. Moreover, since the GA method presents an inherent parallel nature, the computational performance of solving the different problems at the lower level could be improved, as mentioned before.

The GAs are essentially optimization algorithms whose solutions evolve somehow from the science of genetics and the processes of natural selection - the Darwinian principle [16, 17]. As a class of general purpose search methods, the GA approach gives a remarkable balance between exploiting the promising regions of the search space and exploring the search space. They differ from more conventional optimization techniques since they work on whole populations of encoded solutions, called individuals, and each possible solution is encoded as a set of genes.

The most important phases in standard GAs are selection (competition), reproduction (recombination), mutation, and fitness evaluation. Selection is an operation used to decide which individuals to use for reproduction in order to produce new search points. Reproduction or crossover is the process by which the genetic material from two parent individuals is combined to obtain one or more offspring. Mutation is normally applied to one individual in order to produce a new version of it where some of the original genetic material has been randomly changed. Fitness evaluation is the step in which the quality of an individual is assessed. By conducting the search in a global domain, the GA approach reduces the chance of converging to local optima and makes it possible to solve problems involving many parameters. Other advantages of using GA are that it is a self-start method with no special requirement on the initial value of unknown parameters, other than defining a search range, and also it does not need information such as gradients or derivatives of the function to be optimized.

Concerning the wing-box structure, it is a 24 ply GR/EP composite material structure with the following structural properties: $E_{11}=65 \mathrm{GPa}, E_{22}=65 \mathrm{GPa}, \nu_{12}=0.31, \nu_{21}=0.31, G_{12}=6.9 \mathrm{Gpa}$, $\rho=1650 \mathrm{Kg} / \mathrm{m}^{3}$, Tensile strength $\sigma_{1}=527 \mathrm{MPa}$, Tensile strength $\sigma_{2}=490 \mathrm{MPa}$, Tensile strength $\tau_{12}=55 \mathrm{MPa}$, NPLY $=24$, stringers Young's modulus $E=72 \mathrm{GPa}$, stringers mass density $\rho=2800 \mathrm{Kg} / \mathrm{m}^{3}$ and width of stringers $l=3.5 \mathrm{~cm}$. The stacking sequence consists of six sub-groups of layers as follows:

$$
\Theta=\left[\theta_{1} / \theta_{2} / \theta_{3} / \theta_{4} / \theta_{5} / \theta_{6}\right]
$$

where each sub-group $\theta_{k}(k=1, \ldots, 6)$ consists of four layers oriented in the same way. This sequence of angles, considering the range defined in Eq. (30), has been used for encoding the individuals for the GA. Thus each angle represents a gene in the total individual.

In the GA implementation adopted here, the algorithm operates on a fixed-sized population which is randomly generated initially. The members of this population are fixed-length and integer-valued strings that encoded the orientation angles as defined in Eq. (34). The replacement scheme is based on the steady-state approach [18], where a newly created and improved offspring immediately replaces another solution, usually the worst, in the population. The evolutionary operators employed in this work are: Tournament Selection [16, 19], Two-point Crossover [16, 18] and Non-uniform Mutation [19]. 
For the minimization of the objective function $\mathcal{F}_{I I}$, Eq. (29), it has been set a population size of 150 individuals, $20 \%$ mutation rate, $100 \%$ crossover rate and as stopping criteria a number of 400 consecutive generations without improvement of the fittest solution has been adopted.

As far as the static analysis is concerned, it has been performed at thesecond level to calculate the strength of the composite wing-box, and it is based: $(i)$ on applying static aerodynamic loads at a free stream velocity equal to the $80 \%$ of the flutter velocity evaluated at the first level; (ii) on considering the geometric incidence $\alpha_{g}$ constant along the wing-span, $\alpha_{g}=5^{\circ}$, and (iii) on considering the Tsai-Hill criterion applied at the upper ply of the wing-panels as a strength constraint for the composite panel.

Before illustrating the numerical results relevant to the optimization procedure let us see the effect of the anisotropicity parameters $\zeta_{1}$ and $\zeta_{2}$, here considered as independent variables, on the flutter velocity. Fig. 3(a) shows the flutter velocity for a swept wing of $\Lambda=30^{\circ}$ and aspect ratio $\lambda=L / c=7$, where $L$ is the $Y$-wise semi-span, and $c$ is the chord, here considered as a constant along the wing-span. The wing-box height is here $h=14 \mathrm{~cm}$ whereas the thickness of the composite laminate is $h_{l}=4 \mathrm{~mm}$ and the width of the stringers is $a=3.5 \mathrm{~cm}$. As one can observe the flutter velocity changes almost linearly for negative values of $\zeta_{1}$ and for all the values of $\zeta_{2}$ that are in the range of their feasibility. For positive values of $\zeta_{1}$ we observe strong variations on flutter velocity, in particular the relative maximum values of flutter move from positive to negative values of $\zeta_{2}$ as the parameter $\zeta_{1}$ increases.

The above results, obtained for a parametric variation of anisotropicity parameters, cannot be realistic since the values of $\zeta_{1}$ and $\zeta_{2}$ could not be feasible e.g., it does not exist a combination of lamination angles $\theta_{k}$ that satisfies Eqs. (36).

Now let us consider the case of a two-level optimization applied to the same wing of the above example. The wing-box is divided into five equal parts along the wing-span, each part is here considered as a sub-level of the second level optimization procedure as shown in Fig 1(a). For the case under concern there are $5 \times 2$ global design variables $\left(\zeta_{1}\right.$ and $\left.\zeta_{2}\right)$ and $5 \times 6$ local variables $\left(\theta_{k} ; k=1, \ldots, 6\right)$ for a total of 40 design variables.

As far as the second level optimization procedure is concerned and the determination of the angles $\theta_{k}$ of the stacking sequences $\Theta$ different situation have been analyzed according different choices of the angle variation $\Delta \theta_{k}$. In particular results obtained for $\Delta \theta_{k}$ equal to $1^{\circ}, 5^{\circ}, 10^{\circ}, 15^{\circ}, 20^{\circ}, 25^{\circ}$ and $30^{\circ}$ will be discussed. First of all the case of $10^{\circ}$ lay-up sequence variation has been considered.

Fig. 3(b) shows a typical flutter diagram. In this figure the real parts of the first five eigenvalues of the aeroelastic system as function of the velocity for three different values of the optimization cycles are reported. It of interest to observe that aeroelastic instability, which onset when the real part of the eigenvalues become positive (i.e. the aerodynamic damping becomes positive), at the beginning of the optimization is related to the third aeroelastic mode (which in this case is mainly a torsional mode for the wing), whereas at the end of the optimization the second and the third aeroelastic mode become unstable practically at the same value of velocity. This is due the fact that the changing of the anisotropicity coefficients of the composite materials produce a change of the torsional and flexural behavior of the composite wing [6] this in turns affect its aeroelastic stability margins. In this case the "torsional" mode, that initially is unstable at a lower velocity value, approaches during the optimization the "flexural" mode, whereas the second mode (mainly a flexural mode), which at the beginning is "more" stable, approaches the "torsional" mode becoming "less" stable.

Fig. 4(a) shows the flutter velocity vs. the iteration number of the first level optimization. It is possible to observe that: (a) the flutter velocity increases almost parabolically till a value of approximately 300 $\mathrm{m} / \mathrm{s}$ is reached, and (b) when this value is exceeded the increase on the the value of the objective function is very small and practically linear with a very low slope. The explanation for this behavior can be found by observing the trend of the constraints during the optimization process. Figs. 4(b) and 5(a) show the Tsai-Hill conditions, which represent also the constraints for the first level optimization, evaluated at $5 \%, 30 \%, 50 \%, 70 \%$, and $90 \%$ of the wing span.

In particular at the $5 \%$ (Fig. 4(b)) of the wing length a great spread on the numerical values of the Tsai-Hill condition can be observed, which means that as the velocity increases the aerodynamic loads also increase and, since they produce maximum stresses near the clamped end of the wing-box structure, it appears that around the maximum value of the flutter velocity, for some lay-up configurations, the composite wing suffers a failure. At this point the second level optimization based on GA tries to find a lay-up sequence for which the Tsai-Hill failure criterion is not violated. 
The authors have in fact observed that at the second level the GA may find very different lay-up sequences in correspondence to small variations on the parameters $\zeta_{1}$ and $\zeta_{2}$ coming from the first level. Unfortunately the internal interlaminate stresses associated to some of these sequences violate the Tsai-Hill criterion. As a matter of fact with the proposed coordination procedure between the first and the second levels based on Eqs. 31-32 it is possible to force the overall aeroelastic optimization to find the lay-up sequence which satisfy the Tsai-Hill criterion. Fig. 5(b) shows the angular lay-up sequence corresponding to the first wing-box bay for different optimization cycles note the great variations on the angles also in correspondence of the last cycles of the optimization. Tab 1(a) shows the final lay-up sequences for the five wing-span locations.

Table 1: (a) Lay-up sequency for five wing-box sub-section, $\Delta \theta_{k}=10^{\circ}, V_{f}=304 \mathrm{~m} / \mathrm{s}, \Lambda=30^{\circ}, \lambda=7$; (b) Lay-up sequency for five wing-box sub-section, $\Delta \theta_{k}=1^{\circ}, V_{f}=316 \mathrm{~m} / \mathrm{s}, \Lambda=30^{\circ}, \lambda=7$.

(a)

\begin{tabular}{|c|c|}
\hline Sub-level & Lay-up \\
\hline 1 & {$[60 / 50 / 40 / 60 /-20 /-20]$} \\
2 & {$[20 / 30 /-60 / 30 /-50 /-30]$} \\
3 & {$[-90 / 20 /-80 / 40 / 20 / 20]$} \\
4 & {$[90 /-70 / 10 / 0 /-50 /-70]$} \\
5 & {$[-10 /-50 / 80 /-70 /-80 / 20]$} \\
\hline
\end{tabular}

(b)

\begin{tabular}{|c|c|}
\hline Sub-level & Lay-up \\
\hline 1 & {$[58 / 48 /-27 / 77 /-36 /-34]$} \\
2 & {$[-60 /-42 /-67 /-48 / 43 / 30]$} \\
3 & {$[-62 /-45 /-58 /-54 / 26 /-78]$} \\
4 & {$[14 /-78 /-89 / 10 /-74 / 14]$} \\
5 & {$[-70 /-84 /-77 / 11 /-85 /-77]$} \\
\hline
\end{tabular}

It is now of interest to analyze the effects of the angle variations $\Delta \theta_{k}$ applied at the second level on the optimized flutter velocity. Fig. 6(a) shows flutter velocity vs. the cycles of optimization for the case of $\Delta \theta_{k}=1^{\circ}$. The results are very similar to the case of $\Delta \theta_{k}=10^{\circ}$ although the optimized flutter velocity $V_{f}$ is $316 \mathrm{~m} / \mathrm{s}$. This value of $V_{f}$ is $4 \%$ higher than the one obtained when $\Delta \theta_{k}=1^{\circ}$. This result can be easily explained if considering that with a step of $1^{\circ}$ in the search of the best lamination sequence we have in the range $\left[-90^{\circ}, 90^{\circ}\right]$, for each lamina, a number of possible choices, made by the GA, ten times greater than the one relevant to the case of $\Delta \theta_{k}=10^{\circ}$. Tab1(b) shows the relevant lay-up sequence for each of the five wing-box sub-sections.

Similar results were obtained for $\Delta \theta_{k}=5^{\circ}, 10^{\circ}, 15^{\circ}, 20^{\circ}, 25^{\circ}$ and $30^{\circ}$ which are not reported here for sake of brevity. Nevertheless, as summary, in Fig. 6(b) are reported the optimized flutter velocities obtained by varying the lay-up sequences with the above values of $\Delta \theta_{k}$. Furthermore, different wing-box sweep angles were also considered: $\Lambda=0^{\circ}, 20^{\circ}$ and $30^{\circ}$.

It is worth to note that as $\Delta \theta_{k}$ increases the maximum value on the optimized flutter velocity $V_{f}$ decreases. This is true till $\Delta \theta_{k}=15^{\circ}$ once this value is exceeded $V_{f}$ starts to climb again till a value of $\Delta \theta_{k}=25^{\circ}$ after that $V_{f}$ decrease again. It is also interesting to observe that this trend is approximately similar for all the values of the sweep angles here considered. According to the authors this behavior could be explained as combination of two main factors: one related to the periodicity on the values of the parameters $\zeta_{1}$ and $\zeta_{2}$ with the lamination angles (see Eq. 36) and second on the fact that the first level optimization process tries to find the best combinations of $\zeta_{1}$ and $\zeta_{2}$ which gives the maximum value on $V_{f}$ under the condition that all the constraints (especially the structural constraints) are satisfied. As far as the latter condition is concerned, it is clear that with the increasing of $\Delta \theta_{k}$ it may results difficult, for the second level optimization process, to find a combination of the angle-plies orientation that fit both with the values of $\zeta_{1}$ and $\zeta_{2}$ and with the Tsai-Hill conditions.

In particular, the Tsai-Hill conditions result always in contrast with the increase of the flutter velocity. This is due to the fact that the aerodynamic static loads, and consequently the stresses on the composite wing, are evaluated at $80 \%$ of $V_{f}$ as said before. Therefore, as the the flutter velocity goes on to the maximum the Tsai-Hill constraints tend to be violated.

During the optimization it is also possible to highlight two different phases. The first phase is mainly ruled by the first level optimization, in fact the flutter velocity increases very quickly till the quasioptimum value. The second phase is, instead, ruled by the second level optimization or better by the Tsai-Hill constraints here evaluated. This is clearly visible in Figs. 7(a) and 7(b) where flutter velocity and Tsai-Hill constraints are reported versus the optimization cycles for the wing-box with $\Lambda=0^{\circ}$. 


\section{Conclusions}

A two level composite wing-box aeroelastic design optimization has been presented and demonstrated. The procedure is based on a continuous optimization technique used at the upper level (first level) for the determining the optimal anisotropicity parameters, here defined for describing the aero-elastic composite properties of the wing-box. At the second level a discrete optimization based on a genetic algorithm technique was used to determine the composite laminate angles associated to the first level anisotropicity parameters. A communication criterion, based on Tsai-Hill strength condition and on the second level objective functions, was also introduced between the two optimization levels. The results here presented have been shown that this procedure can be used to find a optimal wing design, at least from the flutter analysis here considered.

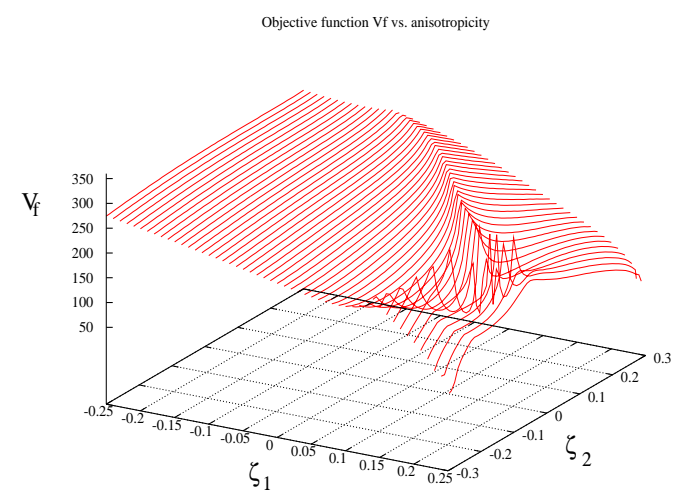

(a)

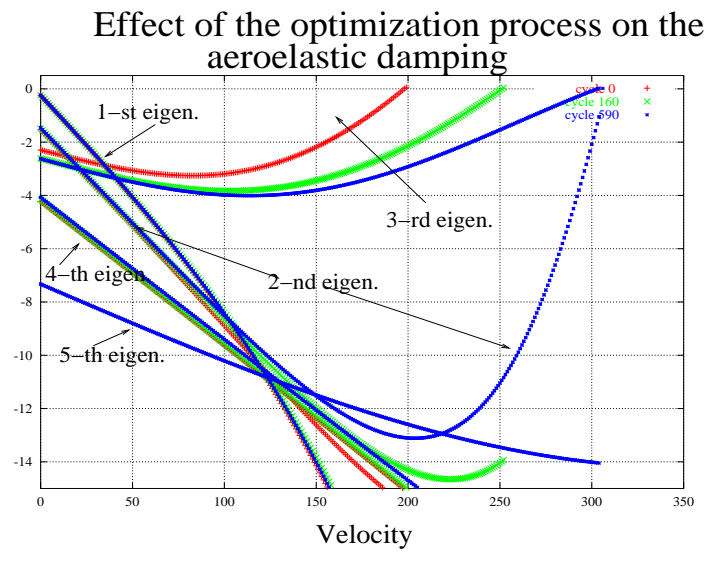

(b)

Figure 3: (a) Flutter Velocity [m/s] vs. anisotropicity parameters: no optimization procedure; (b) Aeroelastic damping coefficients vs. air-velocity at different optimization iterations, $\Lambda=30^{\circ}, L / c=7$.

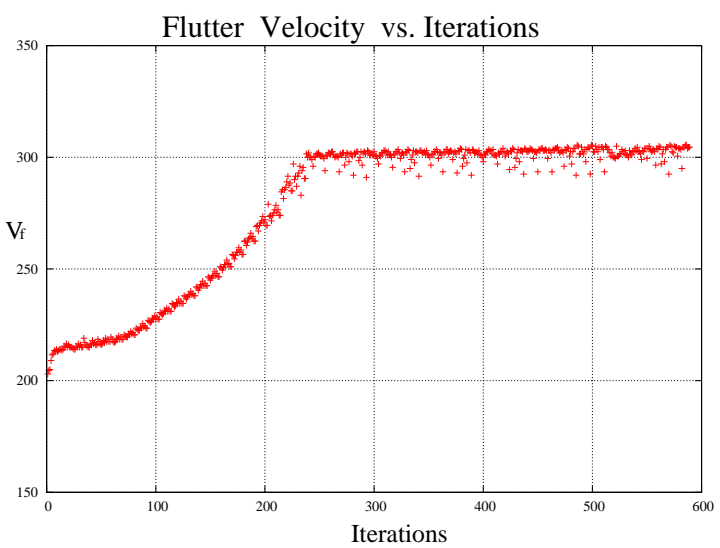

(a)

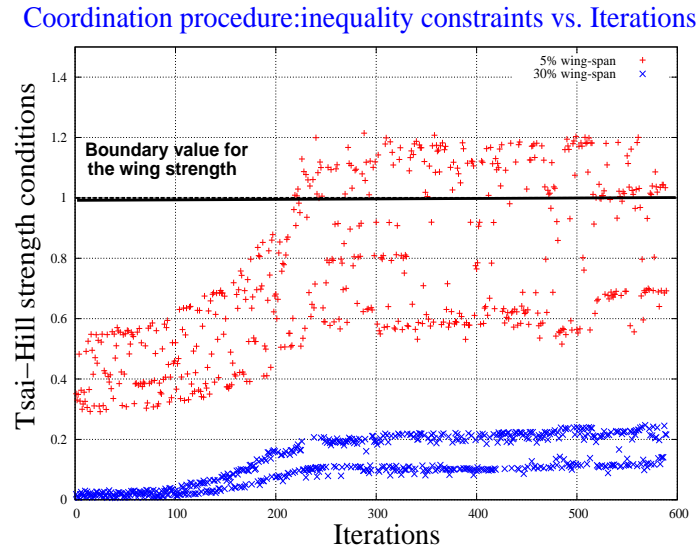

(b)

Figure 4: (a) Flutter velocity $[\mathrm{m} / \mathrm{s}]$ vs. iterations, $\Lambda=30^{\circ}, L / c=7$; (b) Second level optimization: Tsai-Hill coordination conditions vs. cycles, locations 1-2 of wing-span, $\Lambda=30^{\circ}, L / c=7$. 


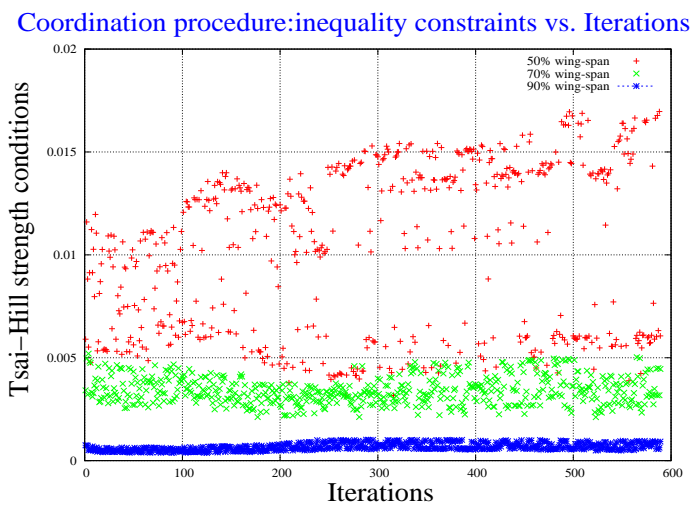

(a)

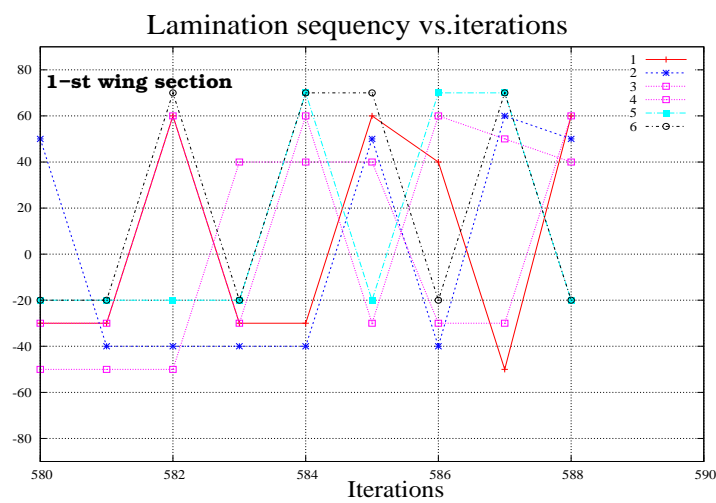

(b)

Figure 5: (a) Second level optimization: Tsai-Hill coordination conditions vs. iterations, locations 3-5 of wing-span, $\Lambda=30^{\circ}, L / c=7$; (b) Lamination sequence vs. iterations, location 1 of wing-span, $\Lambda=30^{\circ}$, $L / c=7$.

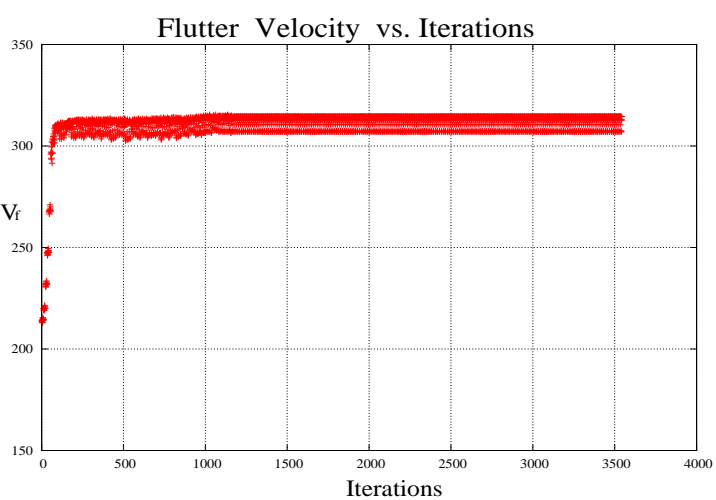

(a)

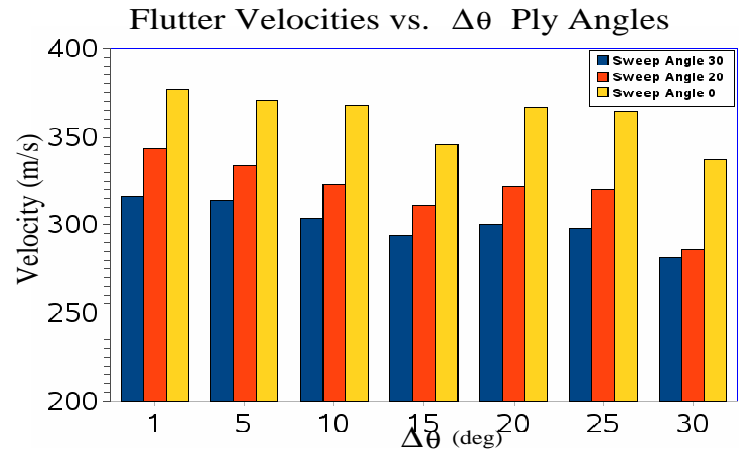

(b)

Figure 6: (a) Flutter velocity $[\mathrm{m} / \mathrm{s}]$ vs. iterations, $\Lambda=30^{\circ}, L / c=7, \Delta \theta_{k}=1^{\circ}$; (b) Flutter Velocities vs. $\Delta \theta$ ply orientation for different Wing-Box Sweep Angles.

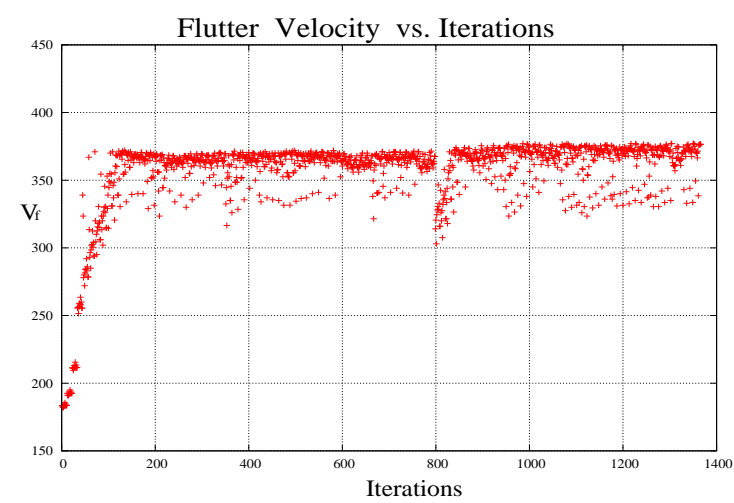

(a)
Coordination procedure:inequality constraints vs. Iterations

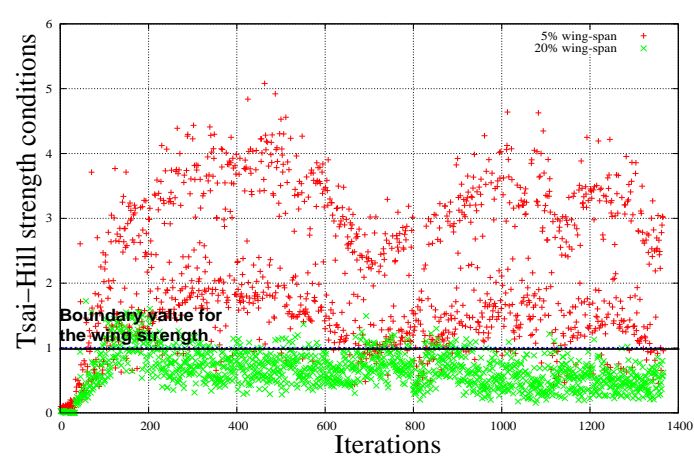

(b)

Figure 7: (a) Flutter velocity $\left[\mathrm{m} / \mathrm{s}\right.$ ] vs. iterations, $\Delta \theta_{k}=1^{\circ}, \Lambda=0^{\circ}, L / c=7$; (b) Second level optimization: Tsai-Hill coordination conditions vs. cycles, locations 1-2 of wing-span, $\Delta \theta_{k}=1^{\circ}, \Lambda=0^{\circ}$, $L / c=7$. 


\section{Appendix A}

In the following are presented the expressions of the global quantities appearing in Eq. (27):

$$
\begin{gathered}
\mathbf{D}^{00}=\sum_{j=1}^{N} \frac{\bar{z}_{j}^{3}-\bar{z}_{j-1}^{3}}{3} \mathbf{H}^{00} ; \\
\zeta_{1}=\sum_{j=1}^{N} \frac{\bar{z}_{j}^{3}-\bar{z}_{j-1}^{3}}{3} \cos 4 \theta_{j} ; \quad \zeta_{2}=\sum_{j=1}^{N} \frac{\bar{z}_{j}^{3}-\bar{z}_{j-1}^{3}}{3} \sin 4 \theta_{j} ; \quad \zeta_{3}=\sum_{j=1}^{N} \frac{\bar{z}_{j}^{3}-\bar{z}_{j-1}^{3}}{3} \cos 2 \theta_{j} ; \quad \zeta_{4}=\sum_{j=1}^{N} \frac{\bar{z}_{j}^{3}-\bar{z}_{j-1}^{3}}{3} \sin 2 \theta_{j} .
\end{gathered}
$$

\section{References}

[1] C.J. He and D.A. Peters, Optimization of Rotor Blades for Combined Structural, Dynamic and Aerodynamic Properties, Structural Optimization, 1992, 5(1-2), 37-44.

[2] J. Walsh, K. Young, J.I. Pritchard, H.M. Adelman and W.R. Mantay, Integrated Aerodynamic/Dynamic/ Structural Optimization of Helicopter Rotor Blades Using Multilevel Decomposition, NASA TP-3465, 1995.

[3] J. Sobieszczanski-Sobieski, B.B. James and A.R. Dovi, Structural Optimization by Multilevel Decomposition. AIAA Journal, 1985, 23(11), 17751782.

[4] G.A. Wrenn and A.R. Dovi, Multilevel Decomposition Approach to the Preliminary Sizing of a Transport Aircraft Wing. AIAA Journal of Aircraft, 1988, 25(7), 632638.

[5] J. Sobieszczanski-Sobieski, Two Alternative Ways for Solving the Coordination Problem in Multilevel Optimization, Structural and Multidisciplinary Optimization, 1993, 6(4), 205-215.

[6] P. Santini and P. Gasbarri, Structural Dynamics of a Cantilever Wing-Like Anisotropic Swept Plate Wings, Journal of Reinforced Plastic and Composite, 2000, 19(14), 1112-1146.

[7] P. Gasbarri, F. Betti, F. Persiani and G.M. Saggiani, Static aeroelastic control of an adaptive wing. Proc. of ICAS 94, 1994, Anaheim, 589-603.

[8] P. Santini, M.A. Sneider and L. Leuzzi, Structural Dynamics of a Cantilever Swept Wing, L'Aerotecnica Missili e Spazio, 1986, 65(3), 141-149.

[9] P. Santini and P.Gasbarri, Lifting Surface in Subsonic Unsteady Regime, Meccanica, International Journal of the Italian Association of Theoretical and Applied Mechanics, 1999, 34(1), 1-27.

[10] J.H. Luo and H.C. Gea, Optimal Orientation of Orthotropic Materials Using an Energy Based Method, Structural and Multidisciplinary Optimization, 1998, 15(3-4), 230-236.

[11] H. Potgeter and N. Stander, The Genetic Algorithm Applied to Stiffness Maximization of Laminated Plates: Review and Comparison, Structural and Multidisciplinary Optimization, 1998, 15(3-4), 221-229.

[12] J.P. Leiva, D.K. Ghosh and N. Rastogi, A New Approach in Stacking Sequence Optimization of Composite Laminates Using GENESIS Structural Analysis and Optimization Software, Proc of 9th AIAA/ISSMO Symposium on Multidisciplinary Analysis and Optimization, 2002, Atlanta, AIAA 2002-5451.

[13] R.T. Haftka and Z. Gürdal, Elements of Structural Optimization, 3rd ed. Boston: Kluwer, 1992.

[14] J.E. Ashton, J.C. Halpin and P.H. Petit, Primer on Composite Materials: Analysis, Westport: Technomic, 1969.

[15] P. Spellucci, A SQP Method for General Nonlinear Programs Using Only Equality Constrained Subproblems, Mathematical Programming, 1998, 82(3), 413-448.

[16] D.E. Goldberg, Genetic algorithms in search, optimization, and machine learning. New York: AddisonWesley Publishing Company Inc., 1989.

[17] J. Holland, Adaptation in natural and artificial systems: an introductory analysis with applications to biology, control, and artificial intelligence, Cambridge, Massachussets: MIT Press, 1992.

[18] G. Syswerda, Uniform crossover in genetic algorithms, Proc. of 3rd International Conference on Genetic Algorithms, 1989, San Mateo, 2-9.

[19] Z. Michalewicz, Genetic algorithms + data structures = evolution programs, Berlin: Springer-Verlag, 1996. 